\title{
Urbanicity, Persecutory Delusions, and Clinical Intervention: The Development of a Brief CBT Module for Helping Patients with Persecutory Delusions Enter Social Urban Environments
}

\author{
Daniel Freeman \\ University of Oxford, $U K$ \\ Helen Waller \\ King's College London, Institute of Psychiatry, UK \\ Ruth Ann Harpur-Lewis and Rosanna Moore \\ Oxleas NHS Foundation Trust, UK \\ Philippa Garety \\ King's College London, Institute of Psychiatry, UK \\ Paul Bebbington \\ University College London, $U K$ \\ Elizabeth Kuipers \\ King's College London, Institute of Psychiatry, UK \\ Richard Emsley and Graham Dunn \\ Manchester University, $U K$ \\ David Fowler \\ University of East Anglia, UK \\ Suzanne Jolley \\ King's College London, Institute of Psychiatry, UK
}

\footnotetext{
Reprint requests to Daniel Freeman, Oxford Cognitive Approaches to Psychosis, Department of Psychiatry, University of Oxford, Warneford Hospital, Oxford OX3 7JX, UK. E-mail: daniel.freeman@psych.ox.ac.uk

(C) British Association for Behavioural and Cognitive Psychotherapies 2013. The online version of this article is published within an Open Access environment subject to the conditions of the Creative Commons AtributionNonCommercial-ShareAlike licence $<$ http://creativecommons.org/licenses/by-nc-sa/3.0/>. The written permission of Cambridge University Press must be obtained for commercial re-use.
} 


\begin{abstract}
Background: Substantial epidemiological research has shown that psychotic experiences are more common in densely populated areas. Many patients with persecutory delusions find it difficult to enter busy social urban settings. The stress and anxiety caused by being outside lead many patients to remain in-doors. We therefore developed a brief CBT intervention, based upon a formulation of the way urban environments cause stress and anxiety, to help patients with paranoid thoughts to feel less distressed when outside in busy streets. Aims: The aim was to pilot the new intervention for feasibility and acceptability and gather preliminary outcome data. Method: Fifteen patients with persecutory delusions in the context of a schizophrenia diagnosis took part. All patients first went outside to test their reactions, received the intervention, and then went outside again. Results: The intervention was considered useful by the patients. There was evidence that going outside after the intervention led to less paranoid responses than the initial exposure, but this was only statistically significant for levels of distress. Conclusions: Initial evidence was obtained that a brief CBT module specifically focused on helping patients with paranoia go outside is feasible, acceptable, and may have clinical benefits. However, it could not be determined from this small feasibility study that any observed improvements were due to the CBT intervention. Challenges in this area and future work required are outlined.
\end{abstract}

Keywords: Delusions, schizophrenia, CBT, urbanicity

\title{
Introduction
}

Treatments for delusions need to be significantly improved. One approach is to use recent advances in understanding their causes to develop more precisely targeted interventions (e.g. Freeman, 2011; Foster, Startup, Potts and Freeman, 2010; Moritz et al., 2011; Waller, Freeman, Jolley, Dunn and Garety, 2011; Myers, Startup and Freeman, 2011; Lincoln, Hohenhaus and Hartmann, 2012). There is also increasing recognition of the need to improve the social recovery of individuals with psychotic experiences (e.g. Fowler et al., 2009). In the current report we describe preliminary work that attempts to link theoretical advances, social recovery, and intervention development.

A substantial epidemiological literature has established that rates of psychosis are higher in urban areas (e.g. McGrath et al., 2004; Sundquist, Frank and Sundquist, 2004; Krabbendam and van Os, 2005; Kelly et al., 2010). This association with urbanicity also applies to milder non-clinical psychotic-like experiences (e.g. van Os, Hanssen, Bijl and Vollebergh, 2001; Scott, Chant and McGrath, 2006), including paranoid thinking (e.g. Johns et al., 2004; Freeman et al., 2011). One hypothesis proposed to explain the link is that a high level of competition enhances the likelihood of social defeat, which then acts as a stressor (Selten and Cantor-Graae, 2005). At a clinical level, patients with persecutory delusions frequently find entering social environments difficult because of their fears of harm. They then avoid going out into busy places in order to minimize threat and the anxious feelings they experience (Freeman et al., 2007). In an experimental study, patients with persecutory delusions who went into a busy urban environment showed increases in anxiety and paranoia compared with staying inside (Ellett, Freeman and Garety, 2008). Anxiety is hypothesized to have a central role in the development of paranoid fears: it causes anticipation of threat, worry, and greater use of safety behaviours (Freeman, 2007). Overall, it is plausible that in outside urban environments it is the presence of many people, the heightened stimulus environment, and the 
stress and anxiety generated that exacerbates paranoia, thereby limiting the social contact of people with psychosis.

On the basis of this epidemiological, theoretical and experimental literature we set out to design a brief intervention specifically focused on helping patients with persecutory delusions feel less distressed when entering busy social urban environments. The aim was to target the anxiety-related cognitive processes that activate the persecutory threat belief. We wished to normalize the stress of urban environments, identify the specific aspects of the environment responsible, highlight how this leads to anxiety and its multiple effects on our minds, bodies and behaviour, and derive strategies to reduce the distress of being outside. We report the first feasibility tests of this intervention, examining the effects of going outside into a busy local street before and after the intervention. We predicted that the intervention would lessen the distress of being outside. A participant group was selected that reported repeated difficulties going outside locally in order to lessen any potential simple habituation effects. However, this initial evaluation of the new intervention did not use a control comparison, meaning that if any clinical change did occur it may not be due to the content of the module.

\section{Method}

\section{Participants}

Eighteen participants were recruited from Oxleas NHS Foundation Trust, the South London and Maudsley NHS Foundation Trust, Camden and Islington NHS Foundation Trust, and Barnet, Enfield and Haringey Mental Health Trust. The inclusion criteria were: a current persecutory delusion meeting the criteria of Freeman and Garety (2000); 50\% or greater conviction in the persecutory belief; reports of anxiety in going outside; aged 18 to 65; and a case note diagnosis of schizophrenia, schizo-affective disorder or delusional disorder. Three suitable participants withdrew before the start of the study (providing no data): one patient developed a physical health condition, one patient began working and couldn't attend appointments, and one patient did not attend the appointments offered.

\section{Design}

Ethical approval was received from the NHS Research Ethics Service. A simple pre and post design was used to test feasibility and acceptability. There was no control group or control condition. In the first session, lasting approximately 90 minutes, the participants were assessed by the clinician, and completed ratings of symptoms before and after going outside into a local busy street on an approximately 10-minutes return trip. The street chosen was dependent upon where the person lived. All patients then received the intervention on a second occasion. This lasted 2-3 hours, but could be split into two meetings. On the immediate completion of the intervention the patients then repeated the walk into the local street, with symptom measures again being taken before and after the exposure.

\section{The intervention}

The intervention (called, "Getting Out and About") (see Figure 1) was presented individually on a computer, with a clinical psychologist present. Patients were encouraged to go through 

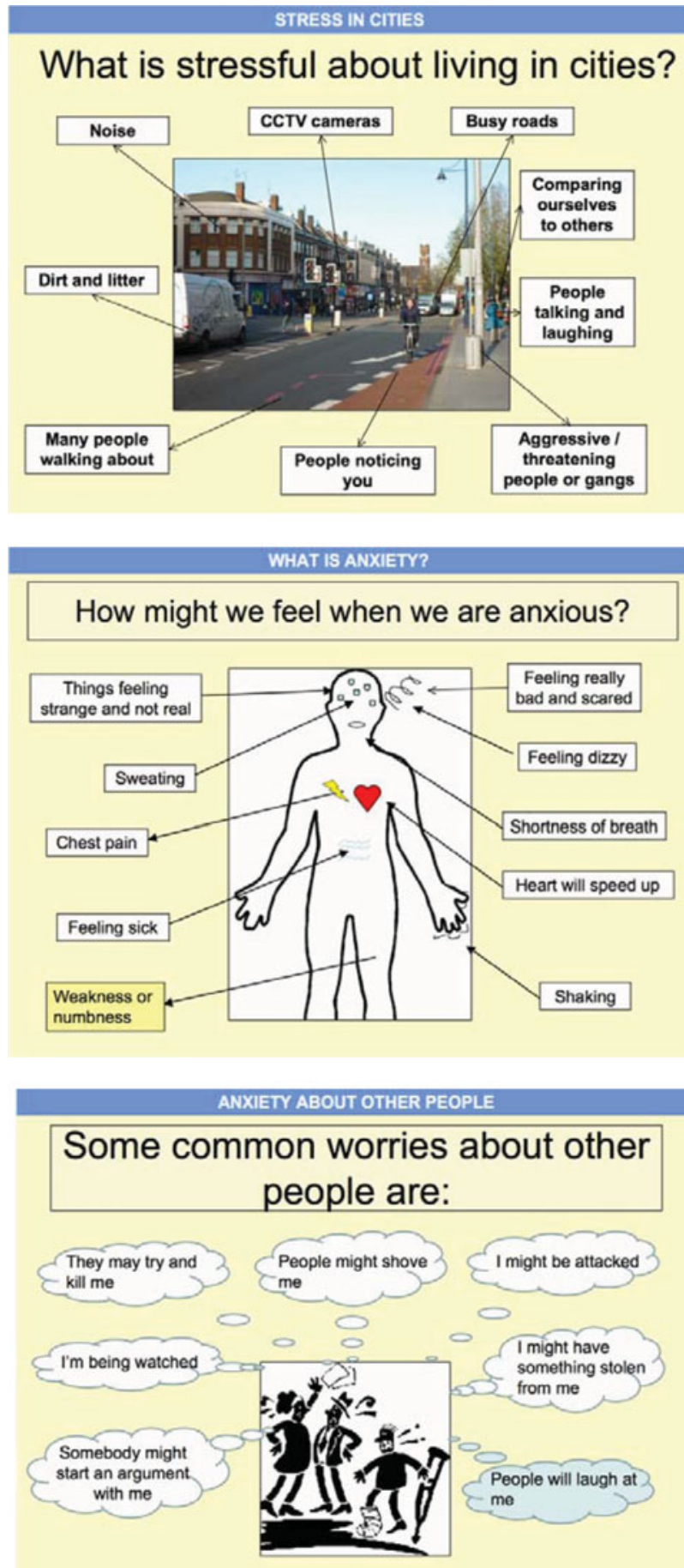

Figure 1. (Colour online) Examples of slides from the intervention module "Getting Out and About" 


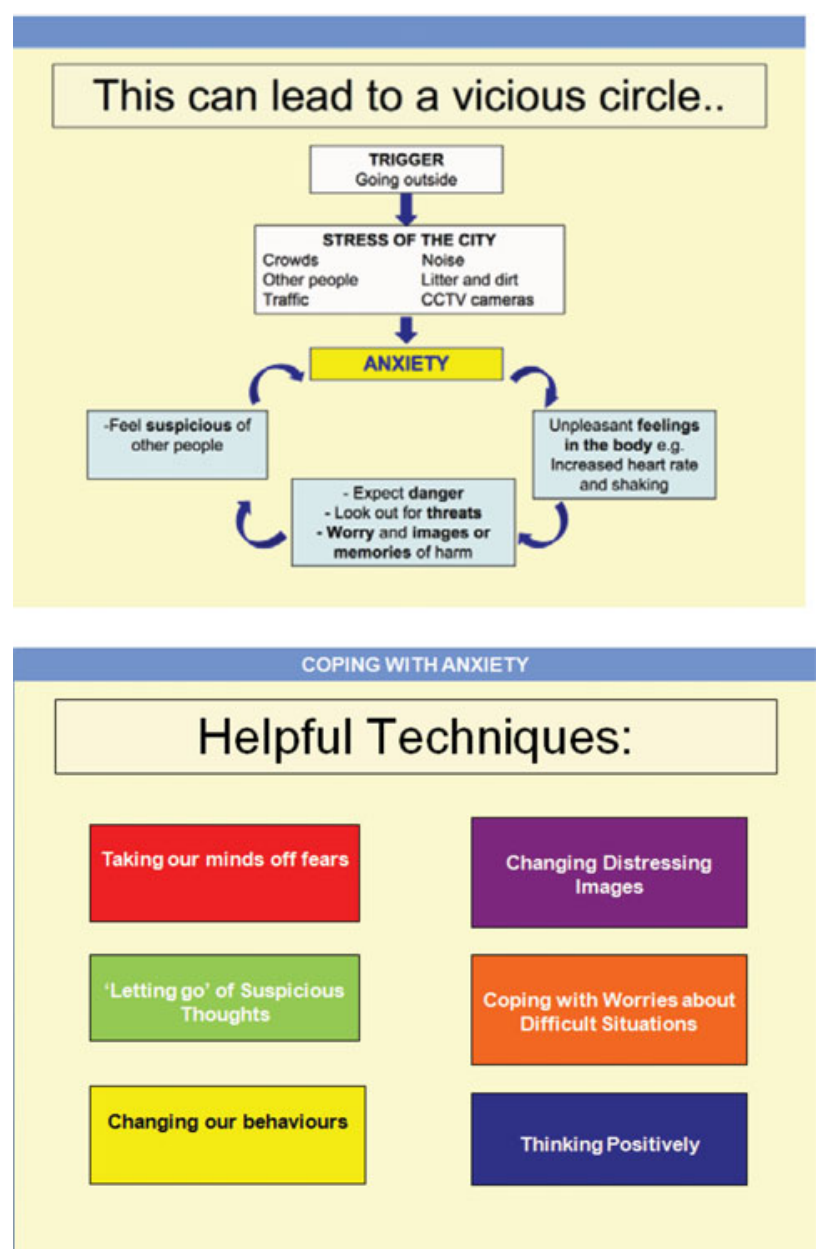

Figure 1. Continued

the programme themselves, stopping for frequent discussion with the therapist. The message of the opening slides was that cities can be stressful and anxiety provoking. A slide with stressful aspects of walking down a street was presented, highlighting, for example, the noise, speeding vehicles, dirt and litter, CCTV cameras, and people walking about. Each aspect could be clicked on the screen to show a picture or video of this aspect of urban environments. The aspects patients found difficult were identified and discussed. The next set of slides highlighted the effects of feeling anxious. This included physiological (e.g. increased heart rate, shortness of breath, feelings of unreality), cognitive (e.g. anticipating threat, worry, focus on threat), and behavioural effects (e.g. a range of safety behaviours including avoidance, escape, keeping vigilant for danger). The specific anxiety effects for the individual patients were discussed. Importantly, the therapist then highlighted how, in this anxious state, fears 
about the intentions of other people would be likely to occur i.e. paranoid thoughts. A simple formulation was then put together. Key boxes linked by arrows were: Going outside, Triggers, Anxiety, Effects of anxiety, Worries about others, Increased anxiety. The summary leading on to the new strategies was:

The trouble is that being outside on the street can make us anxious. The anxiety makes us anticipate danger, focus on threat, worry, and feel in danger. This will lead us to overestimate how much danger we are actually in. We could be safer than we think.

Six strategies were then presented on a menu. The therapist provided brief explanations of each, in order to see which the patient would be most interested in trying. Two or three strategies were then looked through in detail on the computer by clicking on the icon. The six strategies were: 1) Taking our minds off fears (changing focus of attention); 2) Letting go of suspicious thoughts (adopting a mindful approach to fearful thoughts); 3 ) Changing distressing images (imagery work); 4) Coping with worries about difficult situations (decatastrophizing fears); 5) Changing our behaviours (dropping safety behaviours); and 6) Thinking positively (positive self-talk). After a collaborative discussion about how to employ the strategies, patients were then encouraged to try one or two strategies next time they went outside on to the street. A coping card was written as a reminder of the strategy.

\section{Measures}

The assessments were first completed inside, and then repeated outside at the end of the walk in the street. Nine visual analogues scales (0-100) were used to assess how anxious the person was currently feeling, how much they were feeling under threat, the level of paranoid thinking (a mean score from five items: I am being deliberately harmed or upset; I am being followed; There is a conspiracy against me; I am being persecuted; I am being laughed at behind my back), the degree of conviction in the persecutory delusion, and the degree of distress associated with the delusion. The State Social Paranoia Scale (SSPS; Freeman et al., 2007) was also completed before and after going outside. To assess acceptability, three further visual analogue scales were completed at the end of the study assessing on 0 to 10 scales the degree to which participants found the intervention useful, interesting, and enjoyable.

\section{Statistical analysis}

Analyses were carried out using SPSS Version 20.0.0 (IBM, 2011). For the main analysis, change scores from pre to post were calculated for each occasion of going outside (i.e. to determine the effects on one occasion of going into the street), and these were then compared for the two occasions (pre and post intervention) using paired sample $t$-tests. Significance test results for all the analyses are quoted as two-tailed probabilities. We did not alter significance levels for multiple testing, agreeing with the view that "simply describing what tests of significance have been performed, and why, is generally the best method of dealing with multiple comparisons" (Perneger, 1998). 
Table 1. Differences between symptom ratings before and after going outside on each occasion

\begin{tabular}{|c|c|c|c|c|c|c|c|}
\hline Variable & Mean & $S D$ & $\begin{array}{l}\text { Mean } \\
\text { difference }\end{array}$ & $95 \%$ C.I. & $t$ & $d f$ & $p$-value \\
\hline Time 1 Anxiety increase & 20.3 & 29.5 & & & & & \\
\hline Time 2 Anxiety increase & 1.0 & 29.7 & -19.3 & $-44.0,5.4$ & 1.67 & 14 & .117 \\
\hline Time 1 Feeling threat increase & 19.3 & 39.0 & & & & & \\
\hline Time 2 Feeling threat increase & 8.7 & 27.2 & -10.7 & $-33.7,12.4$ & 0.99 & 14 & .338 \\
\hline Time 1 Paranoia increase & 14.7 & 31.3 & & & & & \\
\hline Time 2 Paranoia increase & 2.5 & 18.8 & -12.1 & $-29.3,5.1$ & 1.51 & 14 & .152 \\
\hline Time 1 Conviction increase & 7.2 & 20.6 & & & & & \\
\hline Time 2 Conviction increase & -14.9 & 34.7 & -22.0 & $-48.7,4.6$ & 1.79 & 13 & .097 \\
\hline Time 1 Distress increase & 16.4 & 35.8 & & & & & \\
\hline Time 2 Distress increase & -10.9 & 49.9 & -27.3 & $-52.9,-1.8$ & 2.31 & 13 & .038 \\
\hline Time 1 SSPS increase & 7.4 & 20.4 & & & & & \\
\hline Time 2 SSPS increase & 6.5 & 12.7 & -0.9 & $-7.8,6.0$ & 0.29 & 13 & .775 \\
\hline
\end{tabular}

\section{Results}

\section{The patient group}

The mean age of the participants was $41.4(S D=9.1)$ years old. Nine were male, and six female. The ethnicities of the participants were: White British $(n=9)$, Black British $(n=3)$, and other $(n=3)$. They had diagnoses of schizophrenia $(n=13)$ and schizo-affective disorder $(n=2)$. The mean length of illness was $9.6(S D=11.2)$ years.

\section{Comparison of responses when outside}

It can be seen from Table 1 that on the first occasion of going outside there were increases in symptoms. There was then a uniform and sizeable attenuation of the increase in symptoms at the second time of entering the street scene. For levels of delusional conviction and distress there was a decrease in scores upon going outside on the second occasion. However, the change in symptoms was only statistically significant in relation to the distress associated with the delusion $(p<.05$; effect size $=0.76)$. In other words, the mean increase in distress associated with going outside on the first occasion was reduced significantly on the second occasion.

\section{Acceptability of the intervention}

The mean patient rating of the usefulness of the intervention was 8.7 on the 10 -point scale $(S D=1.5$. minimum $=6$, maximum $=10)$, with seven patients endorsing the top of the scale. The mean patient rating for how interesting the intervention was found was 8.5 ( $S D=$ 1.4 , minimum $=6$, maximum $=10$ ), with six patients endorsing the top of the scale. The mean rating for the enjoyability of the intervention was also favourable, at 7.9 ( $S D=1.9$, minimum $=5$, maximum $=10$ ), with five patients endorsing the top of the scale. 


\section{Discussion}

There is a clinical need to develop interventions that help patients with persecutory delusions feel better able to cope when outside with strangers present. This intervention drew on the theoretical links between urbanicity, stress and anxiety, and paranoia. Being outside in busy urban environments causes stress and anxiety, which are factors linked to the occurrence of paranoid thoughts. The study was an initial feasibility evaluation of the intervention for patients reporting consistent difficulties going outside. Going outside after delivery of the brief intervention was clearly associated with less anxious and paranoid responses. Indeed, after the intervention levels of distress decreased from the rating obtained before going out to the rating obtained after spending time outside (indicating the effects of anticipatory anxiety). However, as there was no control group, the effects cannot be causally attributed to the intervention, and one plausible explanation is that reductions were simply due to the repeat exposure. On the other hand, the patients were entering environments they had been in many times previously with persistent difficulty, and habituation is less likely to occur when safety behaviours remain in place (Salkovskis, 1991; McManus, Scadura and Clark, 2008). Standard CBT for psychosis therefore relies upon behavioural tests of beliefs rather than simple exposure techniques (e.g. Key, Craske and Reno, 2003). The patients themselves viewed the intervention as useful.

The general approach therefore shows promise, and merits a controlled test. However, we also carried out the study in order to learn about the intervention and the methodology of testing it. The patients seemed able to use visual analogue scales both inside and outside in the street, and the scales appeared sensitive to change. However, there was notable variability in symptomatic responses to going outside, even although the group was selected for selfreporting difficulties in such situations. For instance, on the first occasion of going out four patients did not endorse any of the five paranoia items. This might be due to a discrepancy between anticipation and the actual experience (although these four patients also did not endorse the paranoid beliefs before going out). Equally, the patients might have felt safer because of the research context. This clearly weakened the power of the study to find significant effects, but also makes evaluating the outcome of the intervention in this way more difficult. For a larger scale evaluation it may be important to include measures taken outside the research context, for example, examining going out in the flow of daily life using experience sampling methodology (Myin-Germeys et al., 2009) or diary records of the number of times the person has gone out. Multiple different measures can raise the risk of Type 1 error, although the ideal is to see convergence in change across outcome assessments. It would also be an innovation to use mobile technologies to deliver elements of the intervention whilst the person is actually outside (Ben-Zeev, Davis, Kaiser, Krzos and Drake, 2012). We found great interest expressed by the patients in the intervention and its aims, and consider that the length of time spent should be lengthened. It would be valuable to have at least four sessions, which would allow for the trying out of a number of techniques, practice, and consolidation. The patients in the study had typically experienced for many years difficulties going outside and the effectiveness of the intervention in an early episode group would clearly be of interest. In our experience across a number of recent studies (e.g. Foster et al., 2010; Myers et al., 2011), interventions focused on a single outcome and of an acceptable duration can prove both popular and effective with patients with delusions. 


\section{Acknowledgements}

The research was funded by a Wellcome Trust grant (No. 085396). DF is supported by a UK Medical Research Council (MRC) Senior Clinical Fellowship. PG and EK receive support from the Biomedical Research Centre, South London and Maudsley NHS Foundation Trust. There are no conflicts of interest.

\section{References}

Ben-Zeev, D., Davis, K., Kaiser, S., Krzos, I. and Drake, R. E. (2012). Mobile technologies among people with serious mental illness: current use and opportunities for future services. Administration and Policy in Mental Health and Mental Health Services Research. doi: 10.1007/s10488-012-0424-x

Ellett, L., Freeman, D. and Garety, P. A. (2008). The psychological effect of an urban environment on individuals with persecutory delusions: the Camberwell walk study. Schizophrenia Research, 99, 77-84.

Foster, C., Startup, H., Potts, L. and Freeman, D. (2010). A randomised controlled trial of a worry intervention for individuals with persistent persecutory delusions. Journal of Behavior Therapy and Experimental Psychiatry, 41, 45-51.

Fowler, D., Hodgekins, J., Painter, M., Reilly, T., Crane, C., Macmillan, I., et al. (2009). Cognitive behaviour therapy for improving social recovery in psychosis. Psychological Medicine, 39, 16271636.

Freeman, D. (2007). Suspicious minds: the psychology of persecutory delusions. Clinical Psychology Review, 27, 425-457.

Freeman, D. (2011). Improving cognitive treatments for delusions. Schizophrenia Research, 132, 135139.

Freeman, D. and Garety, P. A. (2000). Comments on the content of persecutory delusions: does the definition need clarification? British Journal of Clinical Psychology, 39, 407-414.

Freeman, D., Garety, P., Kuipers, E., Fowler, D., Bebbington, P. E. and Dunn, G. (2007). Acting on persecutory delusions: the importance of safety seeking. Behaviour Research and Therapy, 45, 89-99.

Freeman, D., McManus, S., Brugha, T., Meltzer, H., Jenkins, R. and Bebbington, P. (2011). Concomitants of paranoia in the general population. Psychological Medicine, 41, 923-936.

Freeman, D., Pugh, K., Green, C., Valmaggia, L., Dunn, G. and Garety, P. (2007). A measure of state persecutory ideation for experimental studies. The Journal of Nervous and Mental Disease, 195, 781-784.

IBM (2011). SPSS Statistics Version 20.0.0. Armonk, NY: SPSS, Inc., IBM Company.

Johns, L., Cannon, M., Singleton, N., Murray, R., Farrell, M., Brugha, T., et al. (2004). The prevalence and correlates of self-reported psychotic symptoms in the British population. British Journal of Psychiatry, 185, 298-305.

Kelly, B., O'Callaghan, E., Waddington, J., Feeney, L., Browne, S., Scully, P., et al. (2010). Schizophrenia and the city. Schizophrenia Research, 116, 75-89.

Key, F., Craske, M. and Reno, R. (2003). Anxiety-based cognitive-behavioural therapy for paranoid beliefs. Behavior Therapy, 34, 97-115.

Krabbendam, L. and van Os, J. (2005). Schizophrenia and urbanicity. Schizophrenia Bulletin, 31, 795-799.

Lincoln, T., Hohenhaus, F. and Hartmann, M. (2012). Can paranoid thoughts be reduced by targeting negative emotions and self-esteem? An experimental investigation of a brief compassion-focused intervention. Cognitive Therapy and Research. doi: 10.1007/s10608-012-9470-7 
McGrath, J., Saha, S., Welham, J., El Saadi, O., MacCauley, C. and Chant, D. (2004). A systematic review of the incidence of schizophrenia. BMC Medicine, 2, 13. doi: 10.1186/1741-7015-2-13

McManus, F., Scadura, C. and Clark, D. (2008). Why social anxiety persists: an experimental investigation of the role of safety behaviours as a maintaining factor. Journal of Behavior Therapy and Experimental Psychiatry, 39, 147-161.

Moritz, S., Kerstan, A., Veckenstedt, R., Randjbar, S., Vitzthum, F., Schmidt, C., et al. (2011). Further evidence for the efficacy of a metacognitive group training in schizophrenia. Behaviour Research and Therapy, 49, 151-157.

Myers, E., Startup, H. and Freeman, D. (2011). Cognitive behavioural treatment of insomnia in patients with persecutory delusions. Journal of Behavior Therapy and Experimental Psychiatry, 42, 330-336.

Myin-Germeys, I., Oorschot, M., Collip, D., Lataster, J., Delespaul, P. and van Os, J. (2009). Experience sampling research in psychopathology. Psychological Medicine, 39, 1533-1547.

Perneger, T. (1998). What's wrong with Bonferroni adjustments. British Medical Journal, 316, 12361238.

Salkovskis, P. M. (1991). The importance of behaviour in the maintenance of anxiety and panic: a cognitive account. Behavioural Psychotherapy, 19, 6-19.

Scott, J., Chant, D., Andrews, G. and McGrath, J. (2006). Psychotic-like experiences in the general community: the correlates of CIDI psychosis screen items in an Australian sample. Psychological Medicine, 36, 231-238.

Selten, J.-P. and Cantor-Graae, E. (2005). Social defeat: risk factor for schizophrenia? British Journal of Psychiatry, 187, 101-102.

Sundquist, K., Frank, G. and Sundquist, J. (2004). Urbanisation and incidence of psychosis and depression: follow-up study of 4.4 million women and men in Sweden. British Journal of Psychiatry, 184, 293-298.

Van Os, J., Hanssen, M., Bijl, R. and Vollebergh, W. (2001). Prevalence of psychotic disorder and community level of psychotic symptoms: an urban-rural comparison. Archives of General Psychiatry, 58, 663-668.

Waller, W., Freeman, D., Jolley, S., Dunn, G. and Garety, P. (2011). Targeting reasoning biases in delusions. Journal of Behavior Therapy and Experimental Psychiatry, 42, 414-421. 